$\Rightarrow$ DIABETIC NEPHROPATHY

\section{Restoring podocyte proteostasis in DN}

Impaired podocyte insulin signalling has been shown to promote maladaptive endoplasmic reticulum (ER) signalling in diabetic kidney disease (DKD). Now, Berend Isermann and colleagues report that the cytoprotective coagulation protease activated protein C (aPC) can compensate for defective insulin signalling and restore ER proteostasis in podocytes.

"We previously found that insulin maintains ER homeostasis and thus podocyte function by promoting heterodimerization of $\mathrm{p} 85$ subunits with the ER-dependent transcription factor sXBP1," explains Isermann. "We also showed that aPC conveys strong renal protection independent of blood clotting - in the context of diabetic nephropathy (DN). As aPC signals via protease-activated receptors (PARs) in podocytes, we speculated that targeting aPC-PAR signalling might enable rescue of defective insulin signalling in podocytes and thus protect against DN."

In their recent study, the researchers demonstrate that genetic impairment of PC activation resulted in worsening of the maladaptive renal ER response, impaired nuclear translocation of sXBP1, and increased severity of DKD in mouse models. Conversely, genetic or pharmacological restoration of aPC levels resulted in normalization of ER proteostasis and nuclear SXBP1 levels and protected against DKD. Moreover, chromatin immunoprecipitation sequencing of insulin-stimulated and aPC-stimulated podocytes showed overlapping sXBP1dependent responses involving target genes with roles in the unfolded protein response. "Importantly, these effects were apparent in the absence of additional stimuli, establishing for the first time a physiological function of both insulin and aPC for the regulation of podocyte function," says Isermann.

The researchers conclude that aPC rescues defective insulin signalling and restores proteostasis in podocytes. "A remaining challenge is to identify feasible ways to target aPC-PAR signalling in DN," says Isermann. "Small compounds such as pepducins or parmodulins, which can mimic signalling via G protein-coupled receptor PARs, might constitute a useful approach."

Ellen F. Carney

ORIGINAL ARTICLE Madhusudhan, T. et al. Signal integration at the PI3K-p85-XBP1 hub endows coagulation protease activated protein $\mathrm{C}$ with insulin like function. Blood

http://dx.doi.org/10.1182/blood-2017-02-767921 (2017)

\section{cas}

targeting aPC-

PAR signalling

might enable

rescue of

defective

insulin

signalling in

podocytes and

thus protect

against DN 